\title{
A Hybrid Evolutionary Algorithm for the p-Median Problem
}

\author{
István Borgulya \\ University of Pécs, Faculty of Business \\ and Economics, Hungary \\ H-7621 Pécs, Rákóczi út 80 \\ Tel: 3672501599 \\ e-mail:borgulya@ktk.pte.hu
}

\begin{abstract}
A hybrid evolutionary algorithm (EA) for the p-median problem consist of two stages, each of which is a steady-state hybrid EA. These EAs encode selections of medians as subsets of the candidate sites, apply a recombination operator tailored to the problem, and select symbols in chromosomes to mutate based on an explicit collective memory (named virtual loser). They also apply a sequence of two or three local search procedures to each new solution. Tests e.g. on the benchmark problem instances of ORLIB returned results within $0.03 \%$ of the best solutions known.
\end{abstract}

\section{Categories and Subject Descriptors}

I.2.8 [Artificial Intelligence]: Problem Solving, Control Methods, and Search - heuristic methods

\section{General Terms}

Algorithms.

\section{Keywords}

p-median problem, parallel computing, local search, evolutionary algorithm.

\section{INTRODUCTION}

The p-median problem is the most commonly researched problem in the area of location analysis. In the $p$-median problem there is a set of demand locations and potential facility sites and the aim is to identify the locations of a given number of facilities, $p$, in such a way as to minimize the total distance that demand must traverse to reach its nearest facility. Mathematically, this can be described as follows: Given the set $L=\left\{v_{1}, v_{2}, \ldots, v_{m}\right\}$ of potential locations for the facilities and the set $U=\left\{u_{1}, u_{2}, \ldots, u_{n}\right\}$ of demands, the entries of an $n \times m$ matrix $D=\left(\operatorname{Dist}\left(u_{i}, v_{j}\right)\right)_{n \times m}$ give the distances between $u_{i}$ and $v_{j}$, for all $v_{j} \in L$ and $u_{i} \in U$.

The objective of the $\mathrm{p}$-median problem is to minimize the sum of these distances, i.e.

$$
\operatorname{minimize} f(X)=\sum_{u i \in U} \min _{v j \in X} \operatorname{Dist}(u i, v j)
$$

where $X \subseteq L$ and $|X|=p$. In our interpretation $L$ is equal with $U$.

In practice, the $p$-median problem is used in many areas such as

Copyright is held by the author/owner(s).

GECCO'05, June 25-29, 2005, Washington, DC, USA.

ACM 1-59593-010-8/05/0006. material distribution, transportation, location of industrial plants or public facilities. This problem is $N P$-hard and many heuristics and exact methods have been proposed to solve it.

In this paper we present a hybrid evolutionary algorithm for the pmedian problem, named EPMED (Evolutionary algorithm for the p-median problem).

\section{THE EPMED ALGORITHM}

EPMED has a two stage algorithm structure, where all stages are steady-state hybrid EAs. In the first stage, to speed up the convergence, the algorithm generates new individuals periodically. A new individual is generated the same way as a descendant, but it will increase the number of the population size. Thus, in the first stage new individuals are periodically generated until a maximum number of individuals are completed. In the second stage the algorithm continues the application of the operations of the first stages, but the population-size isn't changed yet (We found similar structure in [3]).

The main functions and characteristics of the EAs are the following:

Representation. We encode selections of medians as subsets of the candidate sites (e.g. $(3,5,19,120)$ by $\mathrm{p}=4)$.

Initial population. The same population and individuals are used in all stages. The first individuals of the $\mathrm{P}$ population are randomly generated. At beginning the population size is less than the maximum population size.

Fitness function. The algorithm uses the objective function $f(x)$ as the fitness function.

Selection operator. In all stages the algorithm selects with tournament selection two different parents.

Recombination operator. The algorithm chooses the two parents with $p_{r}$ probability from the mating pool and the descendent is constructed with a special recombination operator, which is a simpler version of the fusion operator of Beasley [2]. Otherwise it chooses the best parent from the mating pool and the descendent is built by copy-making.

Mutation operator. The algorithm makes some swaps by mutation with $p_{m}$ probability. For a swap the mutation operator selects randomly one of the open facilities and moves this to another site. The new site is also picked randomly from the empty possible places to locate facilities. Only the virtual loser $(V L)$ modifies the random selection of the open facilities: the probability of the selection of an open facility depends on the $V L$ (We choose to adopt the method of [7] that memorises the past failures of evolution through a virtual individual, the virtual loser). 
Local search procedure. A lot of metaheuristics use a variant of the fast interchange local search procedure as a subroutine. E.g. the first is the Variable Neighborhood Search (VNS) [4]. The VNS uses the fast interchange method with a minor difference (We call this variant FIHM). The second is a GRASP version [6], that uses a variant of the fast interchange method too (We call this variant FIRW). The third is the Variable Neighborhood Decomposition Search [5], where the local search method is used in some subspace instead of applying it in the whole solution space (We call this variant $F I D S$ ).

Our algorithm has better performance if we apply more local search procedures to each new solution. So we apply a sequence of two or three local search procedures (CALL FIRW, CALL FIHM or CALL FIRW, CALL FIHM, CALL FIDS). We apply an optimized version of the recombination operator using the FIDS procedure too.

Reinsertion. The algorithm compares the descendent with the best parent. If the descendent is better then the parent, the parent is deleted and the descendent will be a new individual. If the number of the individuals is less then the maximum population size (we increase the number of the individuals), the descendent is inserted into the population without comparison to the parent.

Deleting. In the seconds stage the Deleting procedure deletes a given percentage of the weakest solutions periodically.

Restart procedure. In the second stage if no new best individual in the population is found for more than 50 generations, the EPMED begins the second stage with a smaller population. All individuals except the best $30 \%$ of the population are deleted.

Stopping criteria. The algorithm is terminated if the running time (in CPU seconds) is more than a prefixed time limit.

Table 1. Comparative results for the problems

\begin{tabular}{|l|l|r|l|l|r|l|}
\hline \multirow{2}{*}{$\begin{array}{l}\text { Methods } \\
\text { Tasks }\end{array}$} & \multicolumn{2}{|l|}{ ORLIB } & \multicolumn{2}{l|}{ Koerkel } & \multicolumn{2}{l|}{ fl1400 } \\
\cline { 2 - 7 } & AD & \multicolumn{1}{l|}{ AT } & AD & AT & AD & AT \\
\hline Hybrid & 0.022 & 572 & & & 1.48 & 12479 \\
\hline EPMED & 0.075 & 3981 & 1.72 & 11286 & 10.34 & 32419 \\
\hline VNS & 0.260 & 3135 & & & 3.35 & 27591 \\
\hline TS-2 & 1.420 & 2715 & & & 6.52 & 20650 \\
\hline ADE & 4.400 & 736 & 1.85 & 2373 & & \\
\hline
\end{tabular}

\section{COMPARATIVE TEST RESULTS}

We have tested the EPMED with four sets of test problems: the benchmark set of the ORLIB, the problems from Koerkel [1], two instances from the TSPLIB: the problem fl1400 and the problem pcb3038 (The EPMED was implemented in Visual Basic and ran on a Pentium $41.8 \mathrm{GHz}$ with $256 \mathrm{MB}$ RAM).

As for comparison, we chose different methods: the TS heuristics from [4] (TS_2), the genetic algorithm from [1] (ADE), the VNS by Hansen et al. [4] and a hybrid heuristic from [6] (Hybrid). All method ran on different computers.

Table 1 includes the results of three test-problem sets, and it presents mean values calculated from a number of runs: all problems (tasks) were run 10 times. We give the average relative percentage deviation of the solution from the best known solution (AD), and the average running time to the best solutions (AT) (CPU time in seconds). (The running times do not include the time to compute the all-pairs shortest paths from the graph read and the preprocessing time of the FIRW procedure). Analyzing the values $\mathrm{AD}$ of Table 1 we can confirm that the Hybrid algorithm has the best results, and the VNS has the second best results in general. In the case of the ORLIB problems our method has the second best results.

Both the quality of the results and the running time can be improved using an island model (We used a complete topology between 2-8 processors, chosen the best 1-4 individuals for migrating). The island model for EPMED was simulated on our PC. We tested the model with 10 instances from the fl1400 problem $(10 \leq p \leq 100)$. For comparison, we chose the same methods as earlier (Table 2). We can confirm that the quality of the results (AD) in our island model (with 8 processors) is better as the results of the VNS. Based on this result we hope better results on other test problems too.

Table 2. Comparative results by the island model

\begin{tabular}{|l|l|l|l|l|l|l|l|}
\hline \multicolumn{2}{|c|}{ TS-2 } & \multicolumn{2}{c|}{ VNS } & \multicolumn{2}{c|}{ Hybrid } & \multicolumn{2}{c|}{ EPMED } \\
\hline AD & AT & AD & AT & AD & AT & AD & AT \\
\hline 3.49 & 9001 & 1.33 & 12208 & 1.02 & 880 & 1.09 & 7458 \\
\hline
\end{tabular}

\section{ACKNOWLEDGMENTS}

The Hungarian Research Foundation OTKA T 042448 supported the study.

\section{REFERENCES}

[1] Alp, O., Erkut, E., Drezner, Z.: An Efficient Genetic Algorithm for the $p$-Median Problem. Annals of Operations research 122. 2003. 21-42

[2] Beasley, J.E., Chu, P.C.: A genetic algorithm for the set covering problem. European Journal of Operational Research, 94. 1996. 392-404.

[3] Borgulya, I.: A Heuristics Method for the Quadratic Assignment Problem. Central European Journal of Operations research 11(1) 2003. 3-16.

[4] Hansen, P., Mladenović, N., Perez-Brito, D.: Variable neighborhood decomposition search. Journal of Heuristics. 7(3) 2001. 335-350.

[5] Hansen, P., Mladenović, N.: Variable Neighborhood Search for the p-median. Location Science, Vol.5 No. 4. 1997. 207226.

[6] Resende, M.G.C., Werneck, R.F.: A Hybrid Heuristic for the $p$-Median Problem. AT\&T Labs research Technical Report TD-5NWRCR, 2003.

[7] Sebag, M., Schoenauer, M., Ravisé, C.: Toward Civilized Evolution: Developing Inhibitions. In: Bäck, T. (ed): Proc. of the $7^{\text {th }}$ International Conference on Genetic Algorithm. Morgan Kaufmann Pub. San Francisco, 1997 291-298. 